\title{
Polarization analysis of VLF/ELF waves observed at subauroral latitudes during the VLF-CHAIN campaign
}

\author{
Claudia Martinez-Calderon ${ }^{1 *}$, Kazuo Shiokawa ${ }^{1}$, Yoshizumi Miyoshi ${ }^{1}$, Mitsunori Ozaki ${ }^{2}$, lan Schofield ${ }^{3}$ \\ and Martin Connors ${ }^{3}$
}

\begin{abstract}
Chorus wave emissions are one of the most intense naturally occurring phenomena in the very low (VLF) and extremely low frequency (ELF) ranges. They are believed to be one of the major contributors to acceleration and loss of electrons in the radiation belts. During the VLF Campaign observation with High-resolution Aurora Imaging Network (VLF-CHAIN) from 17 to 25 February 2012, several types of VLF/ELF emissions, including chorus, were observed at subauroral latitudes in Athabasca, Canada. To our knowledge, there has not been any comprehensive study of the physical properties of such emissions at these latitudes. In this study, we calculate spectral and polarization parameters of VLF/ELF waves with high temporal resolution. We found that the polarization angle of several emissions depended on both frequency and time. We suggest that the frequency-dependent events, which usually last several tens of minutes, might be the consequence of the broadening of the ray path that the waves follow from their generation region to the ground. Furthermore, time-dependent events, also lasting tens of minutes, have a polarization angle that changes from negative to positive values (or vice versa) every few minutes. We suggest that this could be due to variations of the wave duct, either near the generation region or along the wave propagation path. Using another ground station in Fort Vermillion, Canada, about $450 \mathrm{~km}$ northwest of Athabasca, we tracked the movements of the ionospheric exit point of three chorus emissions observed simultaneously at both stations. Although we found that movement of the ionospheric exit point does not follow a general direction, it is subject to hovering motion, suggesting that the exit point can be affected by small-scale plasma processes.
\end{abstract}

Keywords: Chorus; Polarization; VLF; ELF; lonospheric source

\section{Background}

There is a variety of emissions that are present in the magnetosphere in the very low (VLF) and extremely low frequency (ELF) range, such as chorus, hiss or quasiperiodic $(\mathrm{QP})$ emissions. They can propagate in whistler mode, and their resonant interactions with energetic particles present in the radiation belts surrounding the Earth are believed to play a very significant role in regulating the dynamics of the outer and inner belts (Helliwell 1967). For example, chorus waves are intense and naturally occurring whistler-mode emissions that originate

\footnotetext{
*Correspondence: claudia@stelab.nagoya-u.ac.jp

1 Solar-Terrestrial Environment Laboratory, Nagoya University, Furo-cho,

Chikusa-ku, 464-8601 Nagoya, Japan

Full list of author information is available at the end of the article
}

near the geomagnetic equator in the Earth's inner magnetosphere. They propagate following the geomagnetic field lines to the ionosphere, then to the ground, where they can be detected (e.g., Helliwell 1967; Sazhin and Hayakawa 1992). Through wave-particle interaction, they can change the energy of relativistic electrons, causing their acceleration (e.g., Bortnik and Thorne 2007; Katoh and Omura 2007; Meredith et al. 2003), or modify their pitch angle, causing their precipitation. Indeed, several studies relate them to micro-bursts of keV-MeV electrons (e.g., Tsurutani et al. 2013; Lorentzen et al. 2001; Saito et al. 2012; Miyoshi et al. 2010) or suggest the contribution of lower and upper band chorus to electron scattering in pulsating auroras (e.g., Nishiyama et al. 2011). Thus, these types of VLF/ELF emissions can make a major contribution to the acceleration and scattering of radiation

\section{是 Springer}

(c) 2015 Martinez-Calderon et al. licensee Springer. This is an Open Access article distributed under the terms of the Creative Commons Attribution License (http://creativecommons.org/licenses/by/4.0), which permits unrestricted use, distribution, and reproduction in any medium, provided the original work is properly credited. 
belt particles (e.g., Inan et al. 1982; Helliwell 1965; Horne et al. 2005; Miyoshi et al. 2013, 2003), making their monitoring necessary to help understand the dynamics of high-energy particles surrounding the Earth.

The region where this interaction takes place, in the magnetosphere's equatorial plane, is usually believed to be directly linked to the point in the lower ionosphere where the waves exit to the ground. However, in some cases, chorus waves can propagate obliquely to the geomagnetic field, from their equatorial source to the plasmasphere (i.e., Bortnik et al. 2009). As such, the study of the spatial and temporal variations of this exit point, which we will call the VLF/ELF chorus ionospheric exit point (e.g., Tsuruda and Hayashi 1975), can yield significant information regarding the wave-particle interaction region and their propagation to the ionosphere (e.g., Lauben et al. 2002).

Most previous studies of VLF/ELF waves at subauroral latitudes have been done using the VLF/ELF Logger eXperiment (VELOX) instrument located in Halley, Antarctica, at $L$-shell 4.3 (e.g., Smith 1995; Smith et al. 1999). Although data has been recorded for the past 20 years, most of the studies did not concentrate specifically on the waves or their physical properties, but were mainly focused on their long-term variation and dependence on the solar cycles. Other studies of chorus wave emissions have been done at higher $L$-shell values, for example, at Syowa Station in Antarctica $(L=6.8)$ using polarization analysis, mainly focusing on full wave analysis and the ionospheric exit point (e.g., Ozaki et al. 2008). However, once again, they did not focus on the physical characteristics.

Consequently, in our study, we perform a spectral and polarization analysis of several types of VLF/ELF emissions, including chorus waves, using the extensive data from the VLF Campaign observation with Highresolution Aurora Imaging Network (VLF-CHAIN). On 17 to 25 February 2012, we installed VLF antennas in Athabasca (ATH, $L=4.3$ ) and Fort Vermillion (FVM, $L=5.4$ ), Canada (i.e., Shiokawa et al. 2014). Our first goal is to obtain spectral and polarization parameters for different types of VLF/ELF emissions by applying the fast Fourier transform (FFT) to the data from ATH. This is, to our knowledge, the first comprehensive study of the physical properties of VLF/ELF emissions using polarization analysis at subauroral latitudes. We then use the data from the second station, FVM, combined with a triangulation method, to roughly determine the ionospheric exit point of three chorus wave events and investigate their motion.

\section{Methods}

The VLF-CHAIN campaign made observations during a 9-day period from 17 to 25 February 2012, using two delta-type loop antennas and all-sky cameras located at the ground stations at the Athabasca University Geospace Observatory $\left(54.60^{\circ} \mathrm{N}, 246.36^{\circ} \mathrm{E}, \mathrm{MLAT}=61.2^{\circ} \mathrm{N}, L=4.3\right)$ and Fort Vermillion $\left(58.38^{\circ} \mathrm{N}, 243.99^{\circ} \mathrm{E}, \mathrm{MLAT}=64.5^{\circ} \mathrm{N}\right.$, $L=5.4$ ), Canada. Both stations are located at subauroral latitudes and are separated by a distance of approximately $450 \mathrm{~km}$. Figure 1 shows their geographic locations, as well as their geomagnetic coordinates, calculated using the 11th International Geomagnetic Reference Field (IGRF11) model with a 2012 epoch time. The antennas are two of the three sets that were developed as part of a VLF observational campaign at Syowa Station (Antarctica) in 2006, so they have similar sensitivities and were calibrated at the same time. Each crossed vertical loop antenna has 10 turns and an area of $16 \mathrm{~m}^{2}$ and provides, in two channels, the north-south (N-S) and east-west (E-W) components of the magnetic field with a sampling rate of $100 \mathrm{kHz}$. More details about this system can be found in Ozaki et al. (2008).

The data from both stations are stored along with two other channels, i.e., the riometer and a GPS time signal that has an accuracy of $1 \mu \mathrm{s}$. The data from ATH is recorded continuously every day, and $9 \mathrm{~min}$ and $50 \mathrm{~s}$ of data is recorded at 10-min intervals. At FVM, only $30 \mathrm{~s}$ of data was recorded during each 10-min time span. The power spectrum density (PSD) of the horizontal magnetic field components was calculated for the N-S and E-W directions ( $\mathrm{CH} 1$ and $\mathrm{CH} 2$, respectively) using the FFT every $0.16384 \mathrm{~s}$, averaging every 16 frequency data points, which corresponds to a frequency resolution of $97 \mathrm{~Hz}$ with the following equation:

$$
P\left(\frac{k}{T}\right)=\frac{1}{T} E\left[X(k) X(k)^{*}\right]
$$

where $P$ is the power spectral density, $k$ is the wavenumber, $T$ is the period, $X$ is the Fourier transform of the signal, $X^{*}$ its conjugate, and $E$ is the ensemble average.

The frequency dependence and the sensitivity of the system have been taken into account by applying a calibration factor and converting the data from $V^{2} / \mathrm{Hz}$ units to $n T^{2} / \mathrm{Hz}$ units. The polarization parameters, i.e., polarization angle, sense, and degree, as well as the coherence between N-S and E-W components of the magnetic field variations, were obtained using the method described by Fowler et al. (1967).

The polarization angle, $\theta$, is the angle between the $\mathrm{N}-\mathrm{S}$ and $\mathrm{E}-\mathrm{W}$ components, defined from the geomagnetic north, where positive angle values are measured westward and negative values eastward. It is calculated using the following equation:

$$
\tan 2 \theta=\frac{-2 \operatorname{Re}\left(P_{x y}\right)}{P_{x x}-P_{y y}}
$$




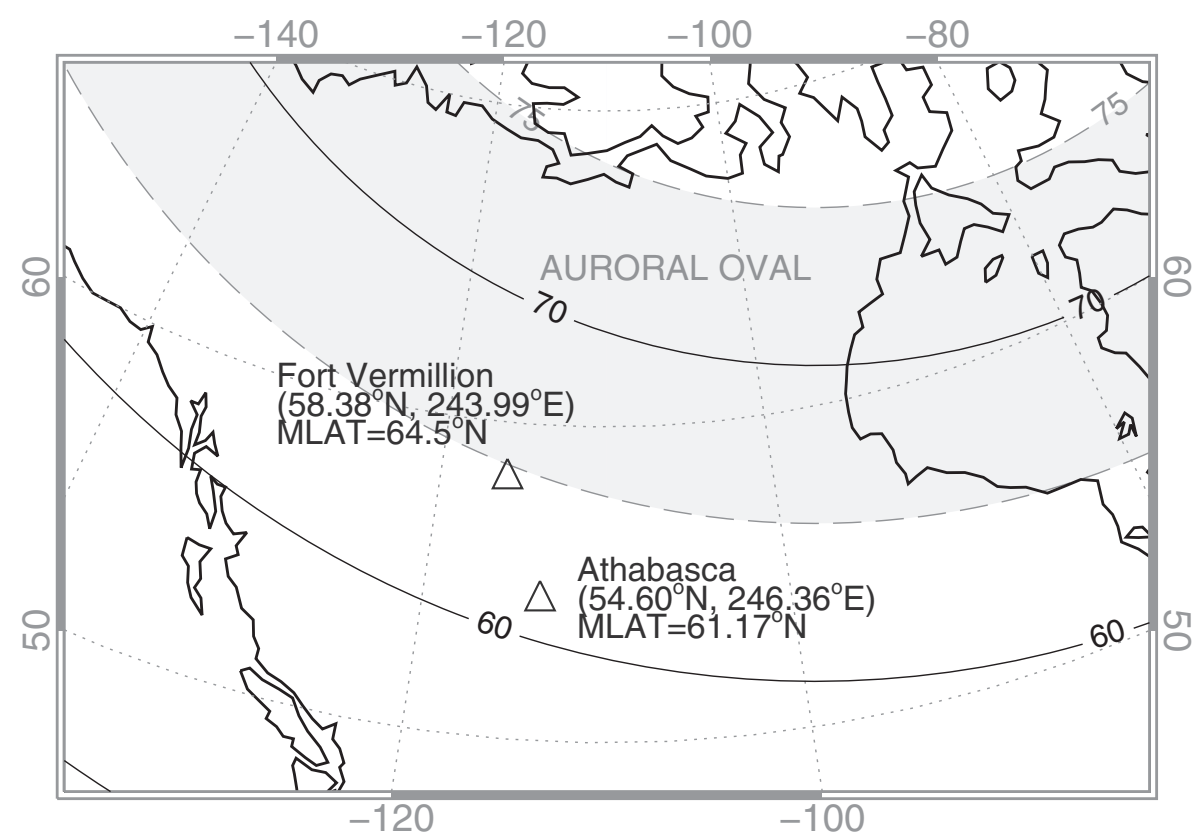

Figure 1 Location of subauroral stations with geographic and geomagnetic latitudes. Map showing the location of the two stations used during the VLF-CHAIN campaign. Dotted lines represent geographic coordinates, and solid lines represent geomagnetic latitudes. The magnetic latitudes (MLAT) were calculated using the IGRF-11 model with an epoch time of 2012.

where $P_{x x}$ and $P_{y y}$ are the power spectrum densities for the N-S and E-W signals, respectively, and $P_{x y}$ is the cross spectrum of the two signals.

The coherence, coh, gives the relationship between N-S and E-W signals, in that the closer the values are to 1, the more similar the signals are, as calculated by:

$$
\operatorname{coh}=\sqrt{\frac{\left|P_{x y}^{2}\right|}{P_{x x} \cdot P_{y y}}}
$$

Finally, the degree of polarization, $R$, oscillating between 0 and 1 , gives the ratio of totally polarized to nonpolarized signals. This gives another indication of the relationship between the N-S and E-W magnetic field components and is calculated using the following equation:

$$
R=\sqrt{1-\frac{4|J|}{\left(P_{x x}+P_{y y}\right)^{2}}}
$$

where $J$ is the coherency matrix giving the polarization of the wave field, written as:

$$
J=\left(\begin{array}{ll}
P_{x x} & P_{x y} \\
P_{y x} & P_{y y}
\end{array}\right) .
$$

Furthermore, we take into account that in the northern hemisphere, waves that are reflected by the ground change their polarization sense from right hand $(\mathrm{RH})$ to left hand (LH). Therefore, in this study, we only consider incoming waves, $\mathrm{RH}$ polarized, meaning that the polarization sense values are between 0 and 1 .

\section{Results}

During the VLF-CHAIN campaign, several interesting features of VLF/ELF emissions were observed, such as QP emissions, falling-tone and rising-tone chorus, hiss, as well as 'bursty-patch' emissions, first reported by Shiokawa et al. (2014). We visually inspected the VLF spectra from ATH from 17 to 25 February, looking for clear chorus, QP, and other emissions, then applied the FFT to obtain spectral and polarization parameters. We then chose the cases with relatively high spectral intensity and coherence values, retaining five instances of risingtone chorus, one falling-tone chorus, four QP emissions, two intense chorus mixed with hiss, and three instances of bursty-patches. The time span of these emissions varied from 1 to 2 min for the bursty-patches to several hours for the other emissions. Using the polarization parameters, we found several interesting characteristics for different types of waves. We first concentrate on the variation of the polarization angle with time and frequency using data from ATH. Then, we pinpoint the source location of three events observed simultaneously at ATH and FVM.

\section{Frequency dependence}

We observed several instances in which the polarization angles of the VLF/ELF emissions were frequencydependent, which we illustrate with the following two examples that include chorus emissions mixed with a QP emission or with hiss: 
(1) On 17 February, there was a long and intense emission that lasted for approximately $4 \mathrm{~h}$, starting around 09:50 UT as a QP emission with rising tones which, after 20 to $30 \mathrm{~min}$, had a chorus-like structure mixed with an intense hiss band ending at around 13:30 UT. This corresponds to post-midnight to early morning, local time (02:50 to 06:30 LT). Figure 2 shows the spectral and polarization parameters of a 30-min time frame at the beginning of this emission. The rising-tone chorus can be seen in the PSD panel from frequencies of approximately 1.5 to $3.5 \mathrm{kHz}$ (Figure 2d), accompanied by some QP structures that can be more clearly observed in the first $5 \mathrm{~min}$. In Figure $2 \mathrm{a}$, high coherence values $(\geq 0.6)$ between N-S and E-W components can be seen for most of the emission around $2 \mathrm{kHz}$, as well as at $3.5 \mathrm{kHz}$ for the first $8 \mathrm{~min}$. Similar results are observed for the polarization degree in Figure 2c. In the polarization angle panel (Figure 2b), we observe that for lower frequencies (approximately $2 \mathrm{kHz}$ ), the angle is negative, approximately $-35^{\circ}$ (blue). However, for higher frequencies (approximately $3 \mathrm{kHz}$ ), the angle is positive, approximately $20^{\circ}$ (light yellow). We also note that in the three polarization panels shown in
Figure 2a,b,c, there is a gap at around $2.8 \mathrm{kHz}$ that is not visible in the PSD panel (Figure $2 \mathrm{~d}$ ).

(2) On 18 February, another long emission, lasting for almost $6 \mathrm{~h}$, was observed. It also started as a QP emission around 14:00 UT, lasting for about $2 \mathrm{~h}$, then became a hiss at around 16:00 UT, mixed with a chorus emission at about 18:20 UT, and finally ended around 20:30 UT. This corresponds to morning to early afternoon, local time (09:00 to 13:20 LT). Figure 3, in the same format as the previous figure, shows a 30-min interval in the middle of the chorus emission. We can clearly identify a hiss band mixed with chorus from 2.5 to $4.0 \mathrm{kHz}$, composed of some rising-tone elements visible around the 8 - to 10-min mark in the PSD panel (Figure 3d). As in the previous example, high coherence and degree of polarization values are observed (Figure 3a,c). In Figure 3b, we can see that the polarization angles at higher frequencies, above 3 $\mathrm{kHz}$, have positive values close to approximately $15^{\circ}$ (light yellow), whereas for frequencies below $3 \mathrm{kHz}$, the angle is negative, closer to approximately $-20^{\circ}$ (light blue).

This frequency dependence of the polarization angle was observed a total of six times during the campaign. It

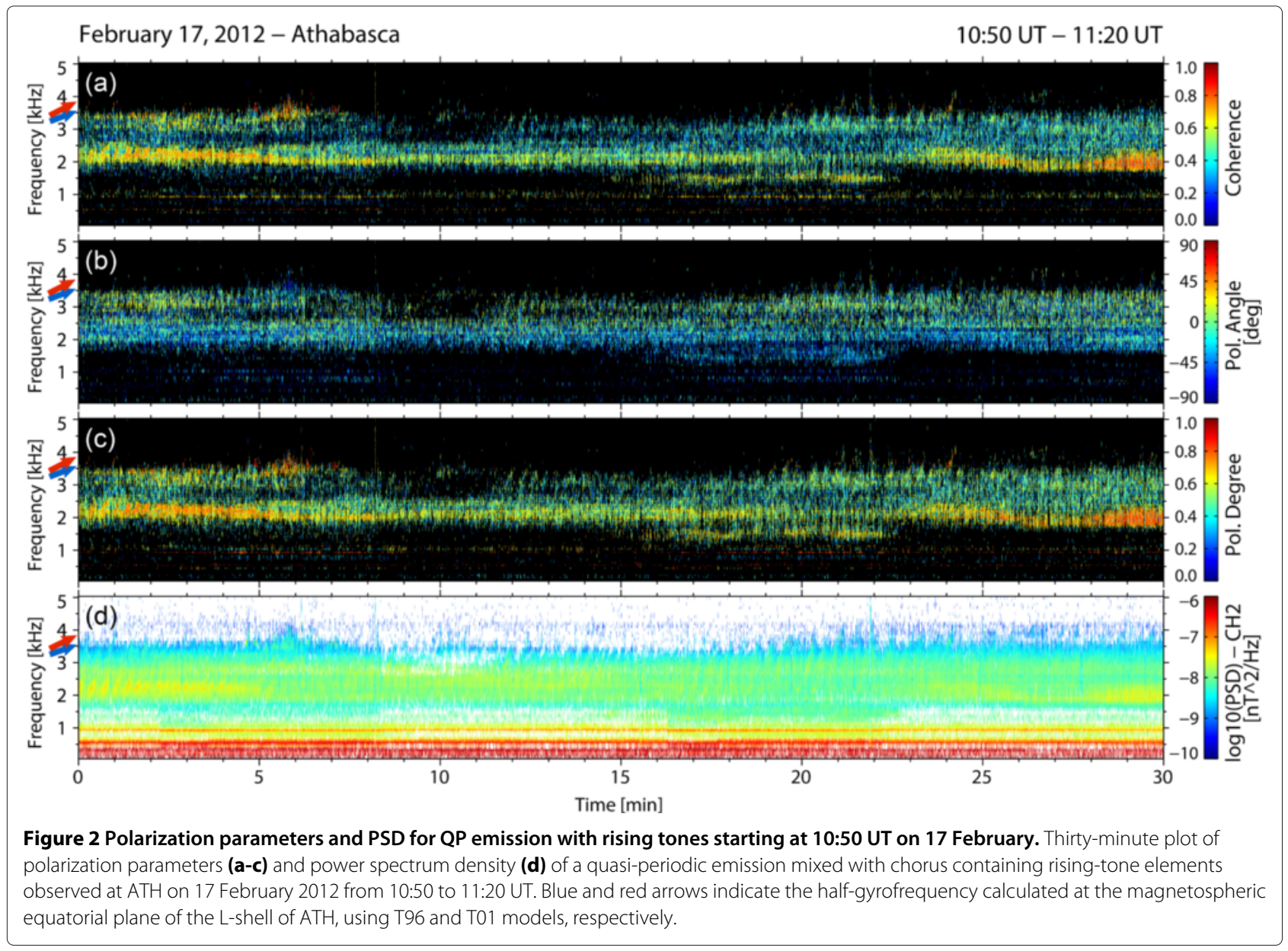




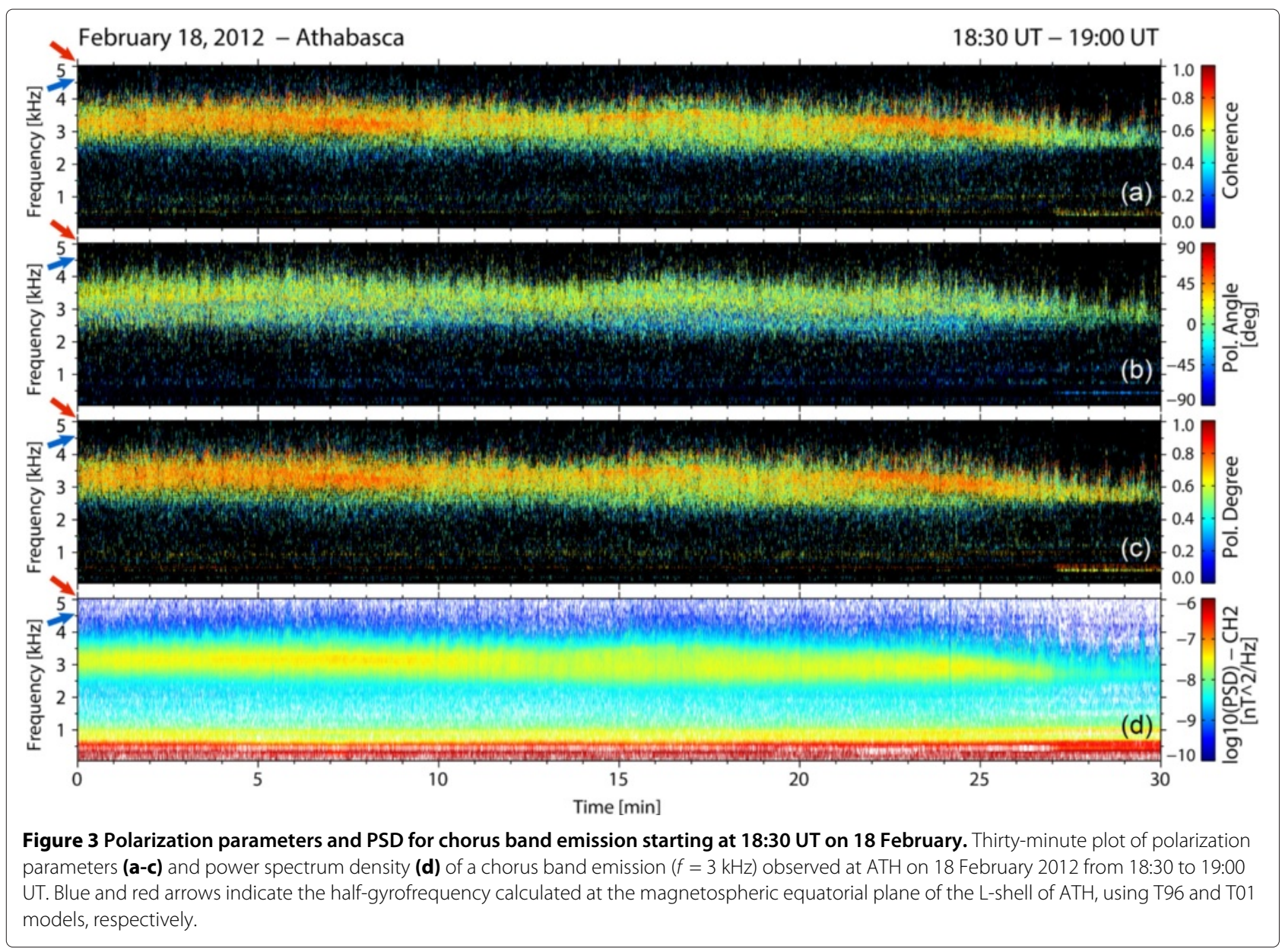

was usually seen once per event, per day (except on 19 February, when it happened twice in 1 day during a particularly long event showing chorus-like structures mixed with QP), lasting from several minutes to several tens of minutes (up to $40 \mathrm{~min}$ ).

\section{Time dependence}

In addition to the frequency dependence of the polarization angle, we noticed several occasions in which the polarization angle of the VLF/ELF emission was timedependent, as illustrated by the next two examples:

(1) On 23 February, we observed an emission suddenly start during a quiet time, lasting for almost $5 \mathrm{~h}$. It started at 16:25 UT with very clear QP elements, quickly showing chorus-like structures, to then mix with hiss and finally disappearing around 21:10 UT, corresponding to morning and early afternoon, local time (09:25 to 14:10 LT). Figure 4d clearly shows the starting point and subsequent $25 \mathrm{~min}$ of the emission for frequencies of 2.5 to $4.0 \mathrm{kHz}$. Figure 4a,c shows high coherence between the N-S and E-W components, as well as high values of degree of polarization (both $\geq 0.8$ ). These panels can help us identify a wave structure with a period of several minutes within the emission, which could be an overlapping QP emission but that only lasts for about $20 \mathrm{~min}$. Figure $4 \mathrm{~b}$ shows that during this 25-min interval, the polarization angle values change from negative (approximately $-30^{\circ}$, blue) to positive (approximately $25^{\circ}$, light yellow), then back to negative (approximately $-40^{\circ}$, dark blue) for another few minutes, only to return to positive values (approximately $20^{\circ}$, light yellow) in a similar time frame. Additionally, we can see the appearance of a gap at around $3.2 \mathrm{kHz}$, which is not detected in the PSD in Figure 4d but visible only in the last 10 to $15 \mathrm{~min}$ of the polarization panels (Figure $4 \mathrm{a}, \mathrm{b}, \mathrm{c}$ ).

(2) On February 19, there was an extremely long QP emission lasting from 14:30 to 23:00 UT, showing rising-tone elements between frequencies of 1 and $2.5 \mathrm{kHz}$. In this case, the emission did not turn into a chorus-like band as in previous examples, but it did show chorus elements during some intervals. Figure 5 shows a 30-min span towards the end of the QP emission: in Figure 5d, we can clearly see the rising tone centered on $1.5 \mathrm{kHz}$, as well as the QP structures with an uneven periodicity of several tens of seconds. Figure $5 \mathrm{a}, \mathrm{c}$ shows high coherence between N-S and E-W magnetic field components and polarization 


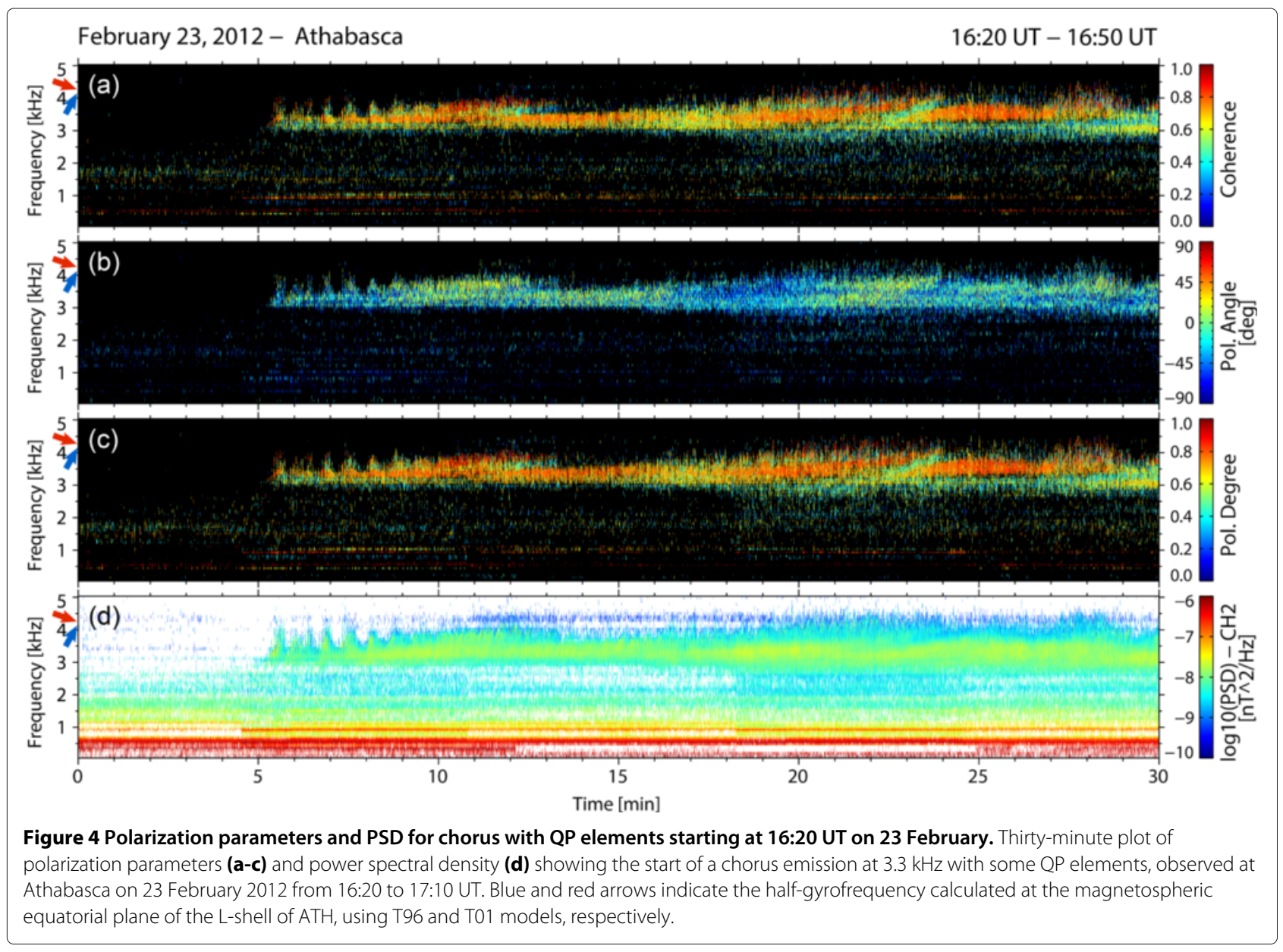

degree values (both $\geq 0.7$ ). In Figure $5 b$, we can see that for the first 6 to $7 \mathrm{~min}$ of this chosen time frame, the polarization angle is negative (approximately $-10^{\circ}$, light blue) or close to $0^{\circ}$ (light green), then changes to positive (approximately $15^{\circ}$, yellow) for about $9 \mathrm{~min}$, only to return to negative (approximately $-40^{\circ}$, dark blue) for a few minutes before finally stabilizing to positive values. Unfortunately, at this point, the intensity of the emissions lessens to a degree that the polarization analysis cannot be properly visualized.

Time-dependent emissions such as the ones shown in these examples, were observed eight times during the 9day period, six of which were found during the very long event on 19 February. In all of the occurrences, intervals of polarization angle sign changes lasted for several tens of minutes, within which sign changes took place every few minutes (from 2 to $10 \mathrm{~min}$ ).

\section{Polarization analysis of bursty-patches}

Shiokawa et al. (2014) reported short time-scale bursty emissions during geomagnetically disturbed periods, observed during the VLF-CHAIN campaign, giving them the name 'bursty-patches'. Figure 6 shows a 10-min interval of the polarization and spectral parameters of the very long event observed on 19 February (also shown as an example in Figure 5), accompanied by a bursty-patch. Figure $6 \mathrm{~d}$ shows the bursty-patch starting at the 8-min mark falling from 5 to $4 \mathrm{kHz}$ for about $1 \mathrm{~min}$, then rising in frequency to slightly above $5 \mathrm{kHz}$ for the next minute or so. In this panel, we can also see a QP emission mixed with chorus with a period of about $20 \mathrm{~s}$, accompanied by rising tones with frequencies of 1.0 to $2.5 \mathrm{kHz}$. Compared to the other examples, Figure 6a shows relatively low coherence (between 0.5 and 0.7), but Figure 6c shows polarization degree values greater than 0.6 . In Figure $6 \mathrm{~b}$, the main chorus emission centered at $2 \mathrm{kHz}$ starts with slightly positive polarization angle values, then, in a few minutes, becomes highly negative (up to $-50^{\circ}$, dark blue). On the other hand, the polarization angle of the bursty-patch remains positive, varying from approximately $5^{\circ}$ (green-yellow) to approximately $20^{\circ}$ (yellow) for its whole duration.

\section{Chorus structures}

With the help of the very high sampling rate of data $(100 \mathrm{kHz})$, we can create high temporal resolution plots of the spectral and polarization parameters that allow us 


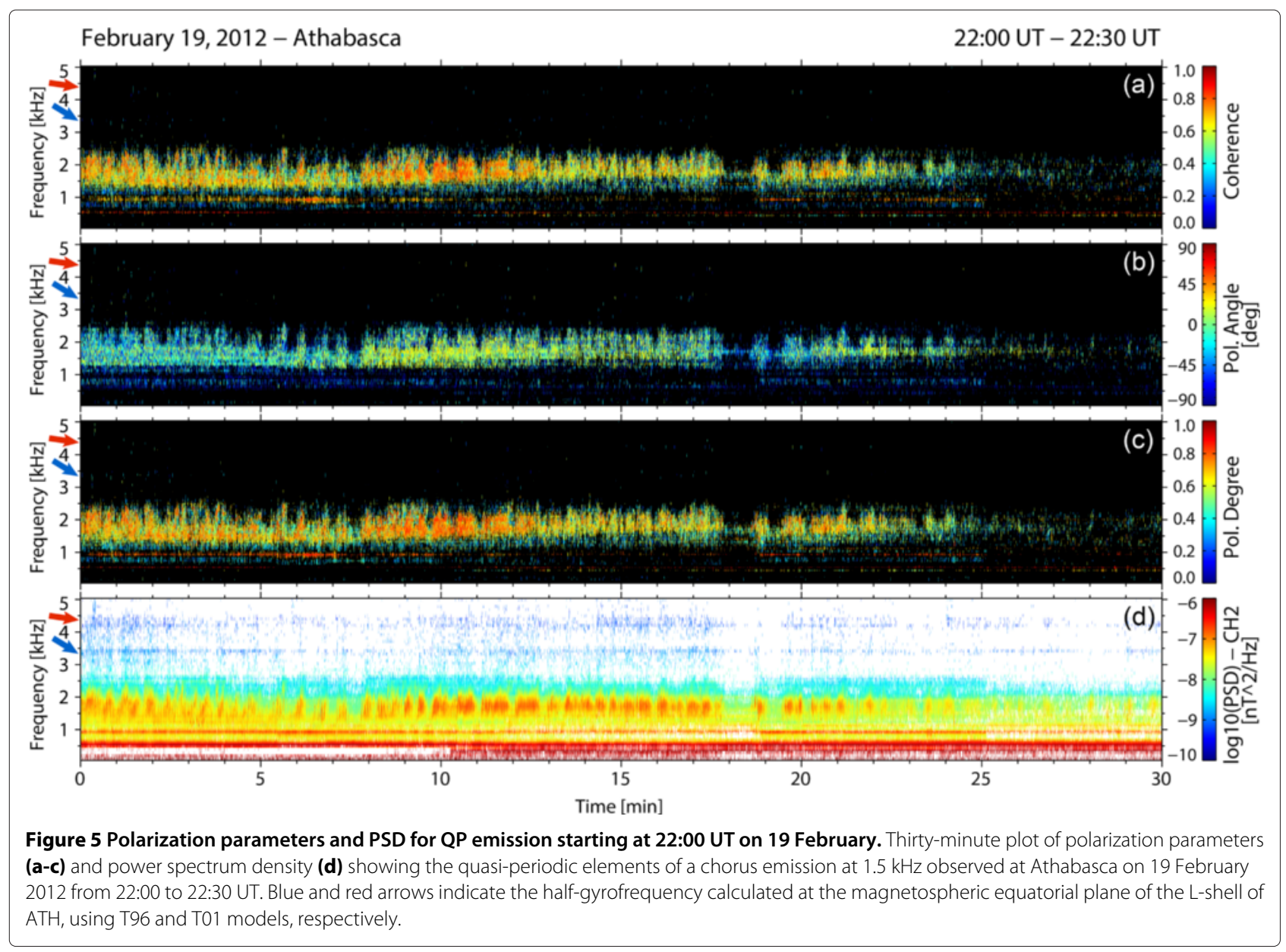

to see the chorus' structure. To improve the temporal resolution from 0.16384 to $0.04096 \mathrm{~s}$, we lowered the frequency resolution from 97 to $388 \mathrm{~Hz}$, averaging every 64 frequency data points. Figure 7 shows a 1-min interval of a chorus emission observed at ATH on 19 February at 18:15 UT, just before noon, local time. In Figure 7d, we can see structures with a periodicity of approximately $10 \mathrm{~s}$, as well as chorus elements with a time scale of a few seconds. Figure 7a,c shows high coherence between N-S and $\mathrm{E}-\mathrm{W}$ components and high polarization degrees, both with values greater than 0.7 . The polarization angle is shown in Figure $7 \mathrm{~b}$. Due to the lowered frequency resolution, however, the detailed view of the variation of the polarization angle for each chorus element is not conclusive. Nonetheless, we can say that the polarization angle for the approximately 10 -s structures seems to be fairly stable, between $0^{\circ}$ (light green) and approximately $20^{\circ}$ (yellow).

\section{Locating the ionospheric exit point}

From 17 to 25 February 2012, we found several chorus events that were simultaneously observed at ATH and FVM. Only three of them were intense enough to yield significant results from the polarization analysis at both stations. The three cases were observed on successive days from 18 to 20 February and at similar times in the early afternoon, local time. Figure 8 shows, in order, from top to bottom, the resulting polarization angle values as a function of time for 18, 19, and 20 February. The raw angle values, each for $30 \mathrm{~s}$ of data per $10 \mathrm{~min}$, are shown in light blue for ATH and orange for FVM. Their calculated mean angle and their corresponding error bars are shown in dark blue for ATH and red for FVM. Among the examples previously shown in the sections 'Time dependence', 'Polarization analysis of bursty-patches', and 'Chorus structures', only the one corresponding to the very long event on 19 February gave satisfying results for both stations (Figure 8b). We observe that all three cases show similar angle variations for both ATH and FVM. This trend can be seen more clearly in Figure 8c, as the angle values for both stations drop until 19:30 UT, then rise at around 20:10 UT, only to drop again until 20:30 UT.

We used the calculated mean value of the polarization angle, combined with triangulation, to follow the movement of the ionospheric exit point during these three events (e.g., Tsuruda and Hayashi 1975, Ozaki et al. 2008). In an effort to make the movement clearer for the reader, 


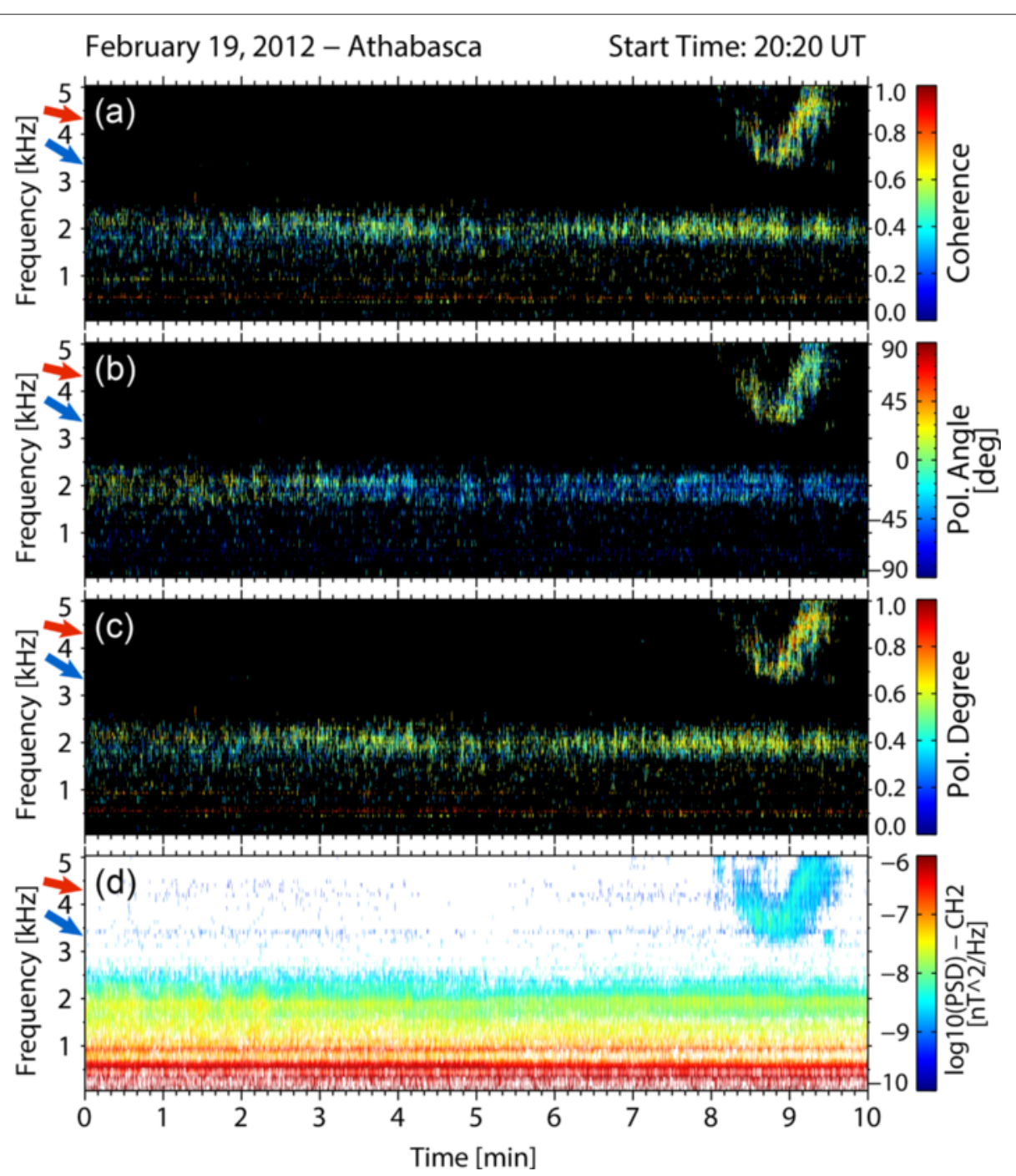

Figure 6 Polarization parameters and PSD for bursty-patch emission starting at 22:00 UT on 19 February. Ten-minute plot of polarization parameters (a-c) and power spectrum density (d) observed at Athabasca on 19 February 2012 from 20:20 to 20:30 UT. This figure shows chorus emission at $2 \mathrm{kHz}$ accompanied by a bursty-patch emission at around $4 \mathrm{kHz}$.Blue and red arrows indicate the half-gyrofrequency calculated at the magnetospheric equatorial plane of the L-shell of ATH, using T96 and T01 models, respectively.

we chose only 4 to 5 relevant points per case, which are plotted in Figure 9. The geographic locations of the two stations and the directions of the incoming chorus waves as seen from ATH are in blue, and those from FVM are in red, for all three events. The numbers inside each dot indicate the progression with time of the location of the ionospheric exit point of the chorus emissions, from 1 to 4 for Figure 9a,c. In the case of Figure 9b, the exit point moved in order, from locations 1 to 4 , then went back to location 1 . The stations are separated by $450 \mathrm{~km}$, so we can estimate that the exit point hovered for a maximum of approximately $90 \mathrm{~km}$ in case (a), approximately $125 \mathrm{~km}$ in case (b), and approximately $275 \mathrm{~km}$ in case (c). We also note that in Figure 9a,b, the ionospheric source of the chorus seems to move in an overall straight line, moving away from ATH or FVM with forward and backward movements. On the other hand, in the case of Figure 9c, the source seems to move in a more disorderly manner, hovering between the two stations, but also in a forward/backward motion. We note that in all three cases, the source location always seems to be between the two stations and closer to FVM than to ATH.

\section{Discussion}

\section{VLF/ELF emissions' characteristics}

Because of the high sampling rate and low-noise environment at $\mathrm{ATH}$, we were able to plot high temporal resolution spectra of different types of emissions, allowing us to see the discrete elements within each chorus emission, as shown in Figure 7 . We cannot see any definite 


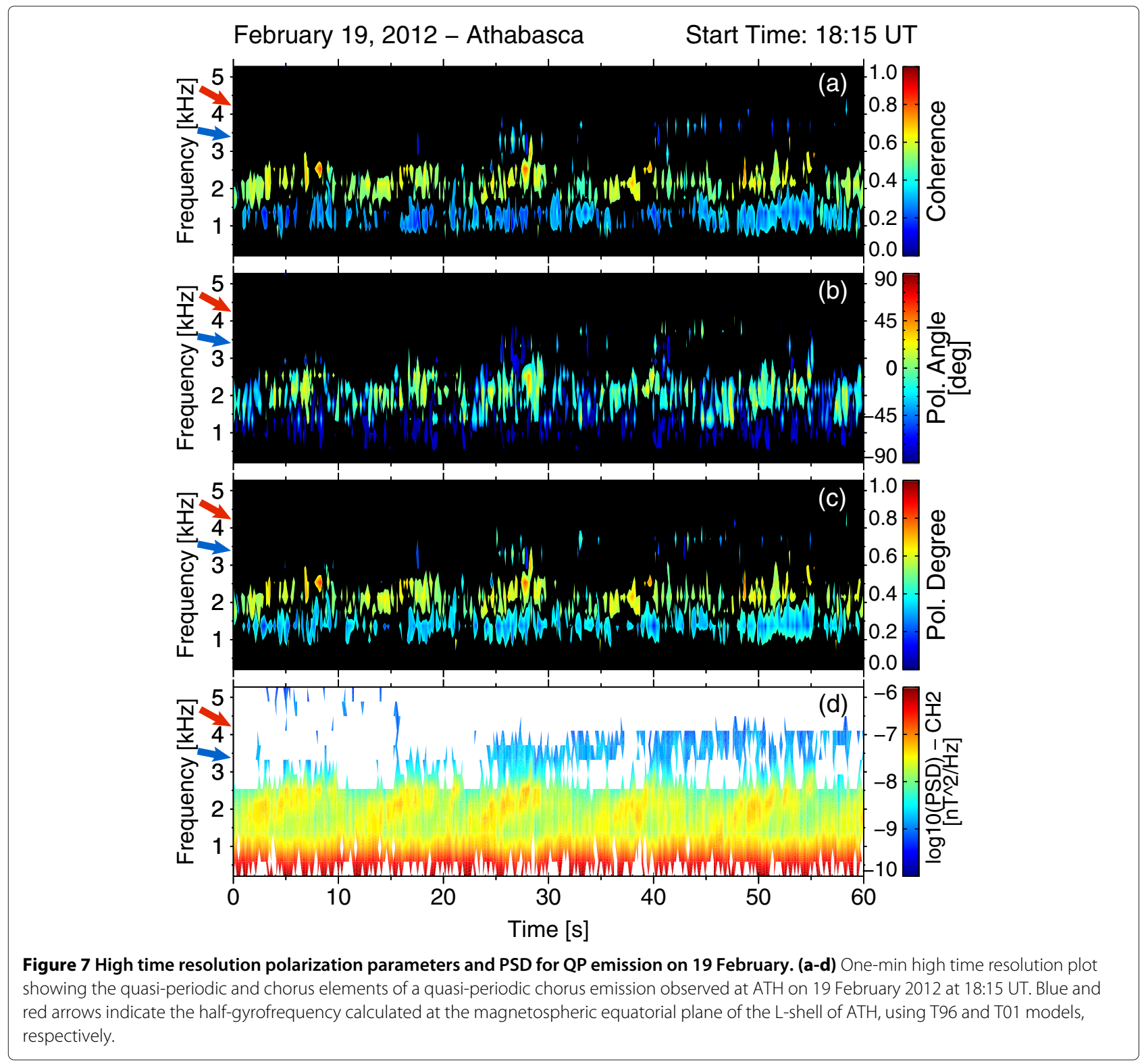

trend or variation of polarization angle within the chorus elements themselves in a time scale of a few seconds or in the structures with time scales of tens of seconds. This suggests that the polarization angle is fairly constant within a single chorus or QP structure, and there is no frequency or time dependence of the polarization angle for small-scale structures.

Nonetheless, on a longer time scale, we found that the polarization angles of several VLF/ELF emissions at subauroral latitudes were frequency-dependent. In the example presented in Figure 2, this frequency dependence could actually be the result of the overlapping of two separate emissions. On a first approach, if we only look at the PSD spectra in Figure 2d, it seems that there is only one emission present extending from 1.5 to $3.5 \mathrm{kHz}$. However, if we look at the coherence and polarization degree panels in Figure 2a,b, we can discern two different emissions: a more intense one centered around $2 \mathrm{kHz}$ and a moderate one at $3.5 \mathrm{kHz}$. It seems that the ground station detected two overlapping simultaneous emissions, each with different and almost opposite polarization angle values, thus suggesting that they exited the ionosphere in two different directions from the station (e.g., Strangeways (1986)). In addition, on closer inspection of the first $5 \mathrm{~min}$ of the PSD panel (Figure 2d), we note that these two emissions are both comprised of rising tones with similar periodicity, on the order of 10 to $15 \mathrm{~s}$. On the other hand, if we look at the three polarization panels, Figure 2a,b,c, we can see a gap at around $2.8 \mathrm{kHz}$ between what could be two emissions. Using the Tsyganenko models T96 (Tsyganenko 


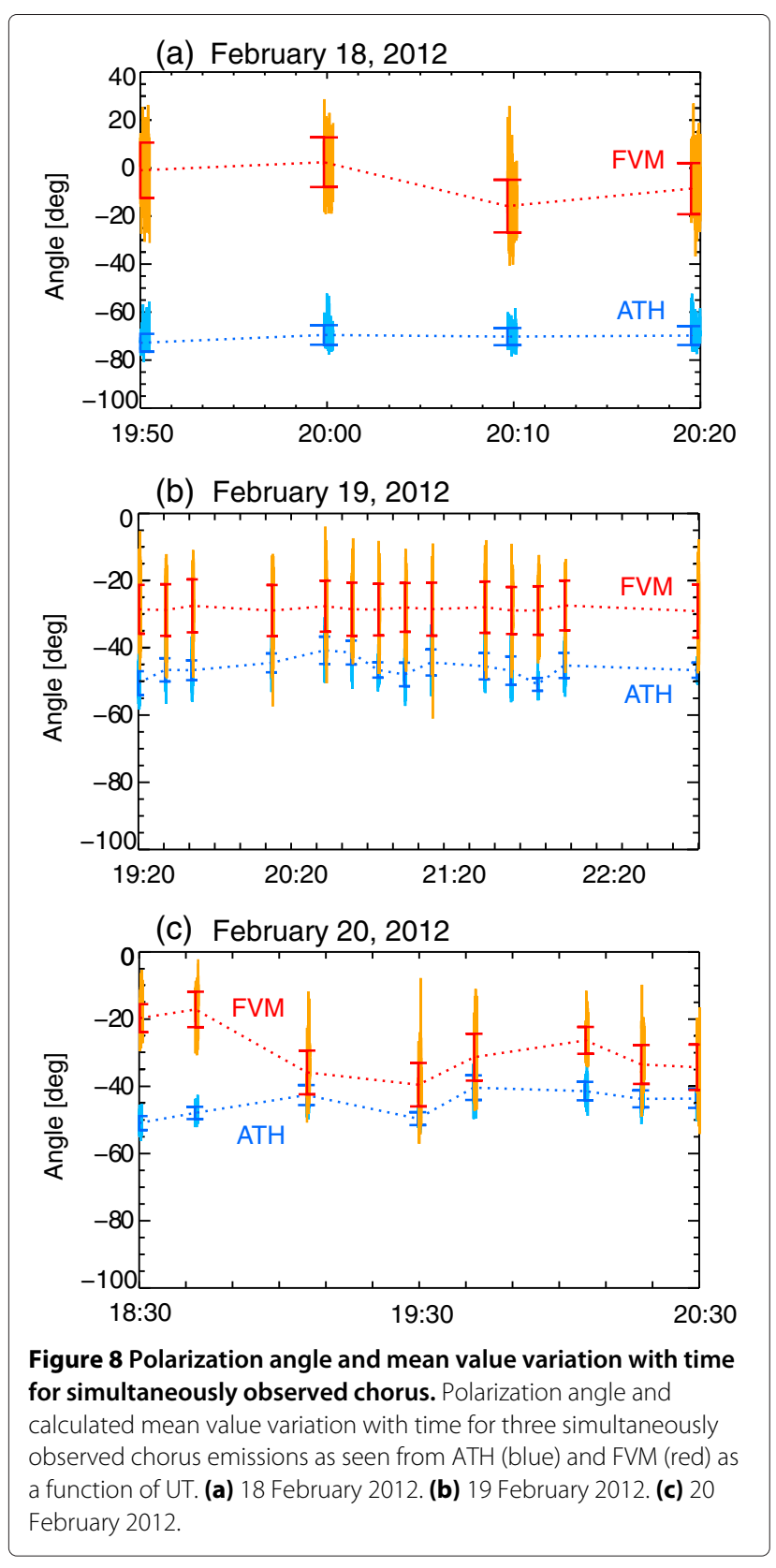

1995 and T01 (Tsyganenko 2002a,b) with solar conditions obtained from the NASA OMNI database, we calculated the value for $0.5 f_{\text {ce }}$ at ATH for all the presented cases. The half-gyrofrequencies obtained with both models are indicated by the colored arrows on the left-hand side of each panel, red for T01 and blue for T96. Their values fluctuate between 3.31 and $5.07 \mathrm{kHz}$, which are somewhat higher than the frequencies in which the gap was observed. Since the gap is present between 2.5 and $2.8 \mathrm{kHz}$, we used the Tsyganenko models to calculate the $L$-shell values corresponding to these values of halfgyrofrequencies and found that they correspond to $4.4 \leq$
$L \leq 4.8$, just between the $L$-shells of the two stations. Therefore, another possible explanation for the different polarization angles observed in this case would be that instead of being two separate emissions, we are actually observing a single chorus emission without a clear gap at the half-gyrofrequency $\left(0.5 f_{\mathrm{ce}}\right)$ as the consequence of nonlinear damping due to the inhomogeneity of the static magnetic field (i.e., Omura et al. 2009). In this case, the emission around $3.5 \mathrm{kHz}$ would correspond to the chorus' upper band, and the one at $2 \mathrm{kHz}$ to the lower band.

Furthermore, we also note that most of the waves are observed below $0.5 f_{\text {ce }}$, suggesting that ATH is only detecting the lower band emission of the chorus. This could be due to the fact that the lower band chorus has been found to be generally more intense than the upper band, covering more $L$-shell values, and thus would be more likely to reach the ground than the upper band (e.g., Meredith et al. 2012). Another possibility is that the wave attenuation in the ionosphere is frequency-dependent or that the propagation from the magnetosphere to the ionosphere depends also on frequency, resulting in the upper band not being able to propagate to the ground. The fact that the gap is located almost $1 \mathrm{kHz}$ below the calculated

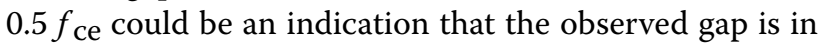
fact due to a combination of two different chorus sources. On the contrary, in the example from 18 February shown in Figure 3, there is no indication that the difference in polarization angle values might be the result of overlapping emissions, and there is also no visible gap. Previous studies (e.g., Yamagishi 1989) have suggested that for non-ducted waves, there might exist a ray path deviation or broadening caused by lower-hybrid resonance. Such mechanisms could explain why the same chorus emissions with different frequencies seem to be observed from the ground as coming from different directions, as shown in Figures 2 and 3.

We also found that the polarization angles of several chorus emissions were time-dependent. Figure 4 shows an example of a time-dependent polarization angle, showing what also seems to be a half-gyrofrequency gap at $3.2 \mathrm{kHz}$. The polarization angle changes value every few minutes, then stabilizes to positive values as the emission 'grows' further after the plotted interval. Following the coherence and polarization degree values in Figure $4 \mathrm{a}, \mathrm{b}$, we can identify what looks like at least three periodic structures in the chorus, spanning several minutes and starting at the 5-, 18-, and 23-min mark. Turning our attention to Figure $4 \mathrm{~b}$, we see that, at least in the first two wave structures, the change of the polarization angle happens during the interval between them. We suggest that this might be the consequence of some localized variation of plasma magnetic field conditions near or at the source of the chorus emission at the geomagnetic equator, signifying the movement of the magnetospheric source region 

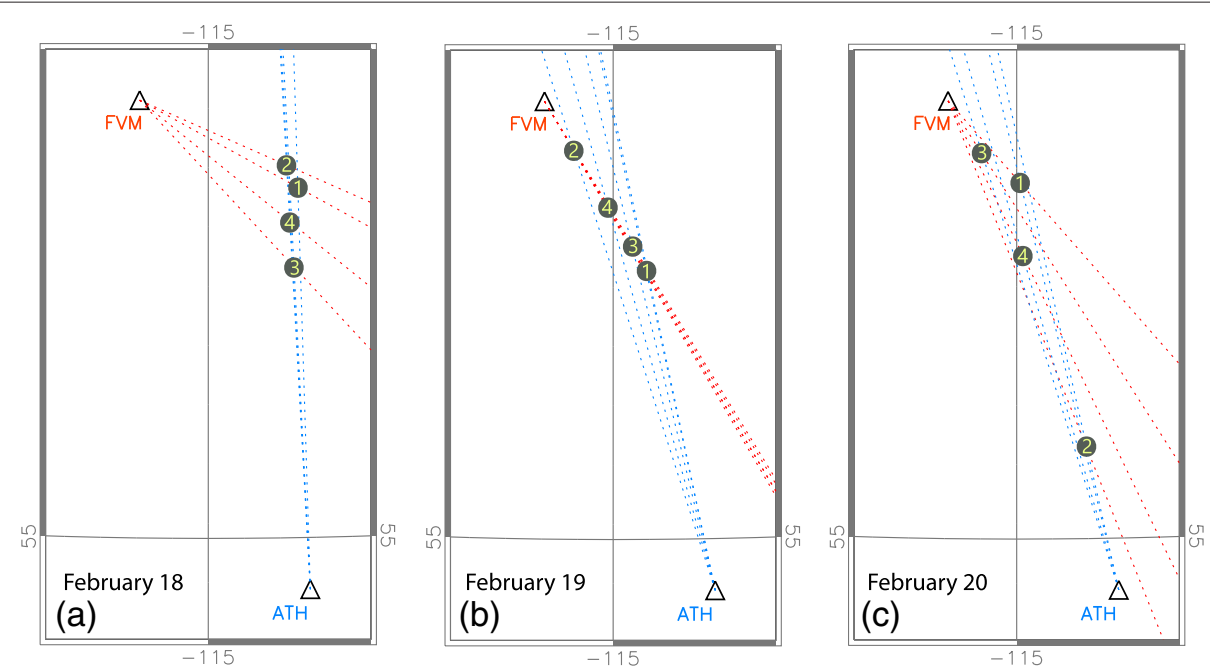

Figure 9 Polarization angle and mean value variation with time for simultaneously observed chorus. Spatial movement with time of the ionospheric exit point for three simultaneously observed chorus emissions detected during three contiguous days at ATH and FVM. (a) 18 February 2012. (b) 19 February 2012. (c) 20 February 2012

with time. Moreover, this figure shows a QP emission that started suddenly during a quiet time, suggesting that this instability at the source could also have been the trigger for this particular chorus emission. At the same time, the other example of time dependence of the polarization angle, shown in Figure 5, does not happen within the structured interval itself but includes a handful of QP elements spanning several minutes at a time. We suggest that this could be the result of the changing wave duct structure between the source in the magnetosphere and the ionospheric exit point of the emission, and not a direct consequence of the movement of the magnetospheric source (e.g., Strangeways (1991)).

It was believed that bursty-patches, first reported by Shiokawa et al. (2014), might be the upper frequency band of chorus emissions. However, as shown in Figure 6b, the main chorus emission and the bursty-patch have almost opposite polarization angle values, the first one being positive (yellow) and the second one negative (dark blue). This suggests that the bursty-patch and the main chorus might come from two separate locations in the ionosphere. However, this could also be explained by a frequency dependence of the ray path of VLF/ELF emissions, including chorus, from their generation region to the ground, as explained previously in this section. Additional study of all the bursty-patches detected during the VLF-CHAIN campaign showed that they usually have positive polarization angle values, between $0^{\circ}$ and $+25^{\circ}$. Even so, at least in one case, we observed the polarization angle value go down to $-10^{\circ}$. In at least two instances, we noticed that the polarization angle of the bursty-patch was independent of the value of the polarization angle of the accompanying main chorus emission. In any case, these results cannot corroborate or disprove that there is a relationship between these two emissions.

\section{Chorus ionospheric exit point}

Using the polarization data from ATH and FVM, we pinpointed the location of the ionospheric exit point of three chorus emissions that were observed simultaneously at both stations. In all three cases, we observed that both ATH and FVM show similar variations of the polarization angle of the chorus emissions, usually negative values (eastward), as shown in Figure 8. This suggests that the chorus emissions detected at these stations are generally exiting the ionosphere at similar points, indicating a preferential path from the magnetosphere to the ground.

Taking into account the angle difference between geomagnetic and geographic north, we plot the incoming direction of the chorus emission as seen by ATH (blue) and FVM (red) for all three cases (Figure 9). The ionospheric exit point of the chorus waves observed simultaneously seems to be located between both stations and slightly closer to FVM than ATH. This reinforces the idea of the existence of a preferred path for the propagation of these waves. Unfortunately, because of the lack of statistics, we cannot give more details about the characteristics of this path. We believe, however, that the proximity of the ionospheric exit point to FVM can be explained by the fact that, because FVM's data tends to be noisier and only recorded for $30 \mathrm{~s}$ every $10 \mathrm{~min}$, a chorus emission seen at ATH might not be intense enough to be detected at FVM. Inversely, if the emission is strong enough to be detected at FVM, it can be more easily detected in the data from ATH. In addition, we note that the movements of the ionospheric source of these chorus emissions seem to follow 
a straight line, as seen from ATH in Figure 9a and FVM in Figure 9b. It is accompanied by backward and forward movements following this general straight direction. However, this direction does not seem to be constant in all three cases, becoming quite dispersed in the third case, as shown in Figure 9c. For this reason, we cannot conclude a fixed preferred direction to which the ionospheric exit point of these chorus waves, and thus their generation region would also move. Even so, because of the hovering motion of the exit point, we suggest that the ionospheric source movement can be altered by small-scale processes with a time scale varying between 20 and $50 \mathrm{~min}$. Consequently, chorus emission generation and their propagation might be also dependent on short time-scale processes.

\section{Conclusions}

Using the high sampling rate of Athabasca data with a low-noise environment, we were able to obtain a closer look at the inner structure of VLF/ELF emissions, including chorus, and follow the variations of the polarization angle for several types of these emissions, including chorus waves, QP emissions, and hiss. Complementing this with the data from Fort Vermillion, we were also able to gather more information concerning the spatial movement of the ionospheric exit point. Applying polarization and spectral analysis during the VLF-CHAIN campaign, we found several cases in which the polarization angles of the VLF/ELF waves at subauroral latitudes were dependent on frequency and time, lasting up to several tens of minutes. We suggest that this might be the consequence of the broadening of the ray path that chorus waves follow from their generation region in the magnetosphere to the ground, causing waves with different frequencies to have different ionospheric exit points. The time dependence of the polarization angle was generally observed several times, lasting for several tens of minutes and changing value from negative to positive (or vice versa) every few minutes. Depending on the observed features of this time dependency, we suggest that this might be due to plasma instability changing either the magnetic field or the electron density near the magnetospheric generation region, or due to modulation of the wave duct between the generation region and the exit point in the ionosphere. The spatial movement of the exit point of VLF/ELF chorus waves could also be observed for three simultaneously observed emissions at ATH and FVM. Although we could not conclude that there was a general drift motion of the ionospheric exit point, we suggest that the movement of this exit point can be affected by smaller scale plasma processes near the source region. A statistical analysis of several types of VLF/ELF waves at subauroral latitudes for a longer period of time, as well as comparison with new satellite data, such as the EMFISIS instrument on board the recently launched Radiation Belts Storm Probes, or using waveform data processing (e.g., Hikishima et al. 2014) in the future Energization and Radiation in Geospace project (i.e., Miyoshi et al. 2012), could yield more insight into the characteristics of the VLF/ELF emissions that were observed in this study.

\section{Competing interests}

The authors declare that they have no competing interests.

\section{Authors' contributions}

CMC carried out the spectral and polarization analysis, visually inspected the data to select the cases presented in this study, calculated the ionospheric exit point through triangulation, and wrote the manuscript. KS, as supervisor of CMC, helped with the discussion, data interpretation, planning, and outline of the manuscript. YM helped with the discussion and data interpretation particularly concerning chorus emissions. $\mathrm{MO}$ calibrated and provided the antenna installed in ATH by KS, IS, and MC. MO also provided information about the antenna at FVM and the handling of its data. IS and MC are responsible for the collection of data in ATH, Canada. MC, YM, and KS also helped with the proofreading of the manuscript. All authors read and approved the final manuscript.

\section{Acknowledgements}

We would like to thank Y. Katoh, H. Hamaguchi, and Y. Yamamoto of the Solar-Terrestrial Environment Laboratory (STEL) at Nagoya University for their skillful support during the observation campaign. The VLF/ELF data is available at STEL's VLF data viewer website accessible at http://stdb2.stelab.nagoya-u. ac.jp/vlf/index2.html. The OMNI data were obtained from the SPDF/GSFC OMNIWeb interface at http://omniweb.gsfc.nasa.gov/form/omni_min.html. We used the instant run for the T96 and T01 models provided by the Community Coordinated Modeling Center (CCMC) accessible at http://swan. gsfc.nasa.gov/requests/instant/tsyganenko.php. Construction and operation of the Athabasca University Geophysical Observatory facilities is supported by the Canada Foundation for Innovation. This work was supported by a Grant-in-Aid for Scientific Research (20244080, 23403009, and 25247080) from the Japan Society for the Promotion of Science, as well as by the Leadership Development Program for Space Exploration and Research at Nagoya University. We also had the support of the Inter-university Upper atmosphere Global Observation NETwork (IUGONET) of the Ministry of Education, Culture, Sports, Science and Technology (MEXT), Japan.

\section{Author details}

${ }^{1}$ Solar-Terrestrial Environment Laboratory, Nagoya University, Furo-cho, Chikusa-ku, 464-8601 Nagoya, Japan. ${ }^{2}$ Kanazawa University, Kakuma-machi, 920-1192 Kanazawa, Japan. ${ }^{3}$ Athabasca University, 1 University Drive, AB T9S 3 A3 Athabasca, Canada.

Received: 28 March 2014 Accepted: 25 November 2014

Published online: 11 February 2015

\section{References}

Bortnik J, Li W, Thorne R, Angelopoulos V, Cully C, Bonnell J, Le Contel O, Roux A (2009) An observation linking the origin of plasmaspheric hiss to discrete chorus emissions. Science 324(5928):775-778

Bortnik J, Thorne R (2007) The dual role of ELFNLF chorus waves in the acceleration and precipitation of radiation belt electrons. J Atmos Sol-Terrestrial Phys 69(3):378-386

Fowler R, Kotick B, Elliott R (1967) Polarization analysis of natural and artificially induced geomagnetic micropulsations. J Geophys Res 72(11):2871-2883

Helliwell RA (1965) Whistlers and related ionospheric phenomena. Stanford University Press, Stanford California, pp. 1-26203316

Helliwell, RA (1967) A theory of discrete VLF emissions from the magnetosphere. J Geophys Res 72(19):4773-4790

Hikishima M, Katoh Y, Kojima H (2014) Evaluation of waveform data processing in wave-particle interaction analyzer. Earth, Planets and Space 66(1):1-10

Horne RB, Thorne RM, Shprits YY, Meredith NP, Glauert SA, Smith AJ, Kanekal SG, Baker DN, Engebretson MJ, Posch JL, Spasojevic M, Inan US, Pickett JS, 
Decreau PME (2005) Wave acceleration of electrons in the Van Allen radiation belts. Nature 437(7056):227-230

Inan U, Bell T, Chang H (1982) Particle precipitation induced by short-duration VLF waves in the magnetosphere. J Geophys Res: Space Phys (1978-2012) 87(A8):6243-6264

Katoh Y, Omura Y (2007) Relativistic particle acceleration in the process of whistler-mode chorus wave generation. Geophys Res Lett 34(13):13102

Lauben DS, Inan US, Bell TF, Gurnett D (2002) Source characteristics of ELFNLF chorus. J Geophys Res: Space Phys (1978-2012) 107(A12):10

Lorentzen K, Blake J, Inan U, Bortnik J (2001) Observations of relativistic electron microbursts in association with VLF chorus. J Geophys Res: Space Phys (1978-2012) 106(A4):6017-6027

Meredith NP, Horne RB, Sicard-Piet A, Boscher D, Yearby KH, Li W, Thorne RM (2012) Global model of lower band and upper band chorus from multiple satellite observations. J Geophys Res: Space Phys (1978-2012) 117(A10):2156-2202

Meredith NP, Horne RB, Thorne RM, Anderson RR (2003) Favored regions for chorus-driven electron acceleration to relativistic energies in the Earth's outer radiation belt. Geophys Res Lett 30(16):1944-8007

Miyoshi Y, Kataoka R, Kasahara Y, Kumamoto A, Nagai T, Thomsen M (2013) High-speed solar wind with southward interplanetary magnetic field causes relativistic electron flux enhancement of the outer radiation belt via enhanced condition of whistler waves. Geophys Res Lett 40(17):4520-4525

Miyoshi Y, Katoh Y, Nishiyama T, Sakanoi T, Asamura K, Hirahara M (2010) Time of flight analysis of pulsating aurora electrons, considering wave-particle interactions with propagating whistler mode waves. J Geophys Res: Space Phys (1978-2012) 115(A10):2156-2202

Miyoshi Y, Morioka A, Misawa H, Obara T, Nagai T, Kasahara Y (2003) Rebuilding process of the outer radiation belt during the 3 November 1993 magnetic storm: NOAA and Exos-D observations. J Geophys Res: Space Phys (1978-2012) 108(A1):3

Miyoshi Y, Ono T, Takashima T, Asamura K, Hirahara M, Kasaba Y, Matsuoka A, Kojima H, Shiokawa K, Seki K, Fujimoto M, Nagatsuma T, Cheng CZ, Kazama Y, Kasahara S, Mitani T, Matsumoto H, Higashio N, Kumamoto A, Yagitani S, Kasahara Y, Ishisaka K, Blomberg L, Fujimoto A, Katoh Y, Ebihara Y, Omura Y, Nosé M, Hori T, Miyashita Y, et al. (2012) The Energization and Radiation in Geospace (ERG) project. Geophys Monograph Ser 199:103-116

Nishiyama T, Sakanoi T, Miyoshi Y, Katoh Y, Asamura K, Okano S, Hirahara M (2011) The source region and its characteristic of pulsating aurora based on the Reimei observations. J Geophys Res: Space Phys (1978-2012) 116(A3):2156-2202

Omura Y, Hikishima M, Katoh Y, Summers D, Yagitani S (2009) Nonlinear mechanisms of lower-band and upper-band VLF chorus emissions in the magnetosphere. J Geophys Res: Space Phys (1978-2012) 114(A7):2156-2202

Ozaki M, Yagitani S, Nagano I, Hata Y, Yamagishi H, Sato N, Kadokura A (2008) Localization of VLF ionospheric exit point by comparison of multipoint ground-based observation with full-wave analysis. Polar Sci 2(4):237-249

Saito S, Miyoshi Y, Seki K (2012) Relativistic electron microbursts associated with whistler chorus rising tone elements: GEMSIS-RBW simulations. $J$ Geophys Res: Space Phys (1978-2012) 117(A10):2156-2202

Sazhin S, Hayakawa M (1992) Magnetospheric chorus emissions: a review. Planet Space Sci 40(5):681-697

Shiokawa K, Yokoyama Y, leda A, Miyoshi Y, Nomura R, Lee S, Sunagawa N, Miyashita Y, Ozaki M, Ishizaka K, Yagitani S, Kataoka R, Tsuchiya F, Schofield I, Connors M (2014) Ground-based VLF/ELF chorus observations at subauroral latitudes - VLF-CHAIN campaign. J Geophys Res: Space Phys 119(9):7363-7379

Smith A (1995) Velox: A new vlf/elf receiver in antarctica for the global geospace science mission. J Atmos Terrestrial Phys 57(5):507-524

Smith A, Freeman M, Wickett M, Cox B (1999) On the relationship between the magnetic and VLF signatures of the substorm expansion phase. J Geophys Res: Space Phys (1978-2012) 104(A6):12351-12360

Strangeways H (1986) Whistler leakage from narrow ducts. J Atmos Terrestrial Phys 48(5):455-462

Strangeways, H (1991) The upper cut-off frequency of nose whistlers and implications for duct structure. J Atmos Terrestrial Phys 53(1):151-169

Tsuruda K, Hayashi K (1975) Direction finding technique for elliptically polarized VLF electro-magnetic waves and its application to the low-latitude whistlers. J Atmos Terrestrial Phys 37(9):1193-1202
Tsurutani BT, Lakhina GS, Verkhoglyadova OP (2013) Energetic electron (>10 keV) microburst precipitation, $\sim 5-15$ s X-ray pulsations, chorus, and wave-particle interactions: a review. J Geophys Res: Space Phys 118(5):2296-2312

Tsyganenko NA (1995) Modeling the Earth's magnetospheric magnetic field confined within a realistic magnetopause. J Geophys Res: Space Phys (1978-2012) 100(A4):5599-5612

Tsyganenko N (2002a) A model of the near magnetosphere with a dawn-dusk asymmetry 2Parameterization and fitting to observations. J Geophys Res: Space Phys (1978-2012) 107(A8):10

Tsyganenko, N (2002b) A model of the near magnetosphere with a dawn-dusk asymmetry 1Mathematical structure. J Geophys Res 107(A8):1179

Yamagishi H (1989) ELF emissions in high latitudes-ray path calculation and ground-satellite observations. Memoirs Nat Inst Polar Res. Ser A, Upper Atmos Phys 19:1-120

\section{Submit your manuscript to a SpringerOpen ${ }^{\circ}$ journal and benefit from:}

- Convenient online submission

- Rigorous peer review

- Immediate publication on acceptance

- Open access: articles freely available online

- High visibility within the field

- Retaining the copyright to your article

Submit your next manuscript at $>$ springeropen.com 